\title{
QPCR and Western Based Fitness Index for Exercised Mice
}

\author{
Zachary Lee ${ }^{1,3}$, Stephanie Vargas ${ }^{1}$, Jordyn Duce ${ }^{1}$, Bradley Schroeder ${ }^{1}$ and John \\ Foley ${ }^{1,2,3}$
}

1) Indiana University School of Medicine, Bloomington, 2) Indiana University School of Medicine, Departments of Anatomy \& Cell Biology and Dermatology, 3) Indiana Clinical and Translational Sciences Institute

Physically fit humans exhibit resistance to infectious and sedentary lifestyle diseases, as well as cancer. This resistance is mediated by alterations in the immune microenvironment. To identify mechanisms, mouse models of exercise have been used. As it has become clear that mice have a natural desire to exercise, voluntary running wheels have become the dominant exercise method eclipsing forced exercise models. To precisely record duration and intensity of exercise, mice are often singly housed with running wheels which induces stress. This project seeks to establish a quantitative molecular and biochemical fitness index for individual mice group housed with access to voluntary running wheels to correlate with disease measures. The gastrocnemius, quadriceps, and soleus muscles were dissected from exercised mice and their controls. These muscles were subject to qPCR for TNNI1 and TNNI2 transcripts, indicators of slow and fast twitch muscles, respectively. Further, the hearts were cut into superior and inferior halves and evaluated by qPCR for the genes NPPA and ATP2A2, indicators of left ventricle thickening. Lastly, a western blot evaluated p70 S6 kinase, another marker of ventricle adaption. Voluntary wheel exercised older mice failed to increase TNNI1 mRNA in the gastrocnemius and quadriceps, indicating the absence of fiber remodeling. In contrast, increased ATP2A2 transcripts were observed in the hearts. Also, increased phosphorylated form of p70 S6 were observed in voluntary exercised mice. Together these findings suggest that voluntary exercise in a group housing setting produces cardiac adaption, but may not induce the changes in skeletal muscle reported with forced exercise. This methodology will be applied to future studies where the impact of voluntary and forced exercise on immune markers and tumor growth are studied. 